\title{
Quantum to classical walk transitions tuned by spontaneous emissions
}

\author{
J. H. Clark, ${ }^{1}$ C. Groiseau, ${ }^{2,3}$ Z. N. Shaw, ${ }^{1}$ S. Dadras $\odot,{ }^{4}$ C. Binegar, ${ }^{1}$ S. Wimberger $\odot,{ }^{5,6, *}$ G. S. Summy,,${ }^{1, \dagger}$ and Y. Liu $\odot^{1, *}$ \\ ${ }^{1}$ Department of Physics, Oklahoma State University, Stillwater, Oklahoma 74078, USA \\ ${ }^{2}$ Dodd-Walls Centre for Photonic and Quantum Technologies, New Zealand \\ ${ }^{3}$ Department of Physics, University of Auckland, Auckland 1010, New Zealand \\ ${ }^{4}$ TOPTICA Photonics Inc., 5847 County Road 41, Farmington, New York 14424, USA \\ ${ }^{5}$ Dipartimento di Scienze Matematiche, Fisiche e Informatiche, Università di Parma, Campus Universitario, 43124 Parma, Italy \\ ${ }^{6}$ INFN-Sezione di Milano-Bicocca, gruppo collegato di Parma, 43124 Parma, Italy
}

(Received 17 August 2021; accepted 4 October 2021; published 22 October 2021)

\begin{abstract}
We have realized a quantum walk in momentum space with a rubidium spinor Bose-Einstein condensate by applying a periodic kicking potential as a walk operator and a resonant microwave pulse as a coin toss operator. The generated quantum walks appear to be stable for up to ten steps and then quickly transit to classical walks due to spontaneous emissions induced by laser beams of the walk operator. We investigate these quantum to classical walk transitions by introducing well-controlled spontaneous emissions with an external light source during quantum walks. Our findings demonstrate a scheme to control the robustness of the quantum walks and can also be applied to other cold atom experiments involving spontaneous emissions.
\end{abstract}

DOI: 10.1103/PhysRevResearch.3.043062

\section{INTRODUCTION}

Quantum walks (QWs) have been actively studied in many experimental systems, such as photons, lattice-confined atoms, and trapped ions, since the first theoretical model was introduced in 1993 [1-7]. Possessing spin degrees of freedom, spinor Bose-Einstein condensates (BECs) have also been suggested as ideal candidates for QW implementation [8]. Two important components of QWs are a walk operator to shift a walker in positions or momentum space and a coin toss operator to determine the direction that the walker shifts in each step [9]. In this work, a rubidium spinor BEC subjected to a series of periodic optical pulses, which can be described as an atom-optics kicked rotor, is utilized to create a $\mathrm{QW}$ in momentum space [10-13]. These periodic pulses construct one-dimensional optical lattices and act as a walk operator in momentum space. Resonant microwave pulses, entangling two hyperfine spin states, are the coin toss operator. In contrast to classical random walks with Gaussian distributions, QWs distribute ballistically because atoms conducting QWs can be in a superposition state $[1,10,11]$. Other advantages of QWs studied in this paper include hitting target points faster than classical walks, fast propagation, and entanglement between internal and external degrees of freedom $[1,14]$. QWs thus

\footnotetext{
*sandromarcel.wimberger@unipr.it

$\dagger$ gil.summy1@gmail.com

‡yingmei.liu@okstate.edu
}

Published by the American Physical Society under the terms of the Creative Commons Attribution 4.0 International license. Further distribution of this work must maintain attribution to the author $(s)$ and the published article's title, journal citation, and DOI. have many proposed and realized applications in various research fields including quantum information, metrology, and topological phenomena [15].

In this work, we demonstrate that quantum walks generated in momentum space can be stable for up to 10 steps and then quickly transit to classical walks due to spontaneous emission (SE) induced by the laser beams imprinting a momentum change. The SE effects have been observed in our previous experiments and are pervasive in other experiments utilizing atom-optics kicked rotors [16-18]. In principle, the SE effects could be inherently present in most experiments involving a quantum system and the impact of these effects on quantum measurements increases as the duration of the experiment increases. To better understand the SE effects, we investigate the SE-tuned quantum to classical walk transitions by introducing well-controlled SE events with an additional laser which does not interfere with the kick or shift laser used in our QW experiments. Those effects are manifold since SE acts as projective measurement in the internal electronic spin degree of freedom of the atom. On the other hand, SE has the twofold effect on the external center-of-mass degree of freedom of the atoms in our BECs: First, it changes the quasimomentum and hence the conditions of being in the QW or not, see Refs. [10,11], and, second, it biases the QW toward the direction of the ground state into which the electronic degree is projected. This is also contrary to previous experiments [16] with just one effective internal state in which SE only had an influence on the quantum-resonance condition and hence on the external degree of freedom. In our experiments, the probability of a SE event and the induced decoherence appear to increase with the evolution of time, i.e., with the number of steps in a $\mathrm{QW}$. We also confirm the SE events lead to a biased momentum distribution, which agrees well with our numerical simulations. Our findings demonstrate a scheme to control the robustness 
of quantum walks and can also be applied to other cold atom experiments involving spontaneous emissions $[19,20]$.

\section{THEORETICAL PREDICTIONS}

Similarly to our previous works, we describe each QW step with an operator $\hat{\mathbf{U}}_{\text {step }}=\hat{\mathbf{T}} \hat{\mathbf{M}}[10,11]$. A unitary walk operator $\hat{\mathbf{T}}$ implemented by atom-optics kicked rotors entangles the internal (i.e., spin) and external degrees of freedom, which leads to a momentum change of $p_{m}=m \hbar P_{0}$ [10-13]. Here $P_{0}$ is the wave vector of the one-dimensional lattice, $\hbar$ is the reduced Planck's constant, and $m$ is an integer number. The coin operator $\hat{\mathbf{M}}$, created by a microwave pulse resonant with the transition between $\left|F=1, m_{F}=0\right\rangle$ and $\left|F=2, m_{F}=0\right\rangle$ states of ${ }^{87} \mathrm{Rb}$ atoms, produces a superposition of these two internal states. We apply a controlled amount of SE during a QW sequence using an independently controlled laser, which excites atoms from the $|F=2\rangle$ ground state resonantly to the $\left|F^{\prime}=3\right\rangle$ excited state. The laser coupling $\Omega=\gamma \sqrt{\frac{I}{2 I_{s}}}$ is small compared to the excited state hyperfine splitting between $\left|F^{\prime}=3\right\rangle$ and $\left|F^{\prime}=2\right\rangle$, so that the $|F=2\rangle \rightarrow\left|F^{\prime}=2\right\rangle$ transition can be assumed to be too far detuned to create a significant population in $\left|F^{\prime}=2\right\rangle$. Here $I$ is the intensity of the laser, $I_{s}$ is the saturation intensity, and $\gamma$ is the decay rate [21]. Due to selection rules, the atom can only decay from $\left|F^{\prime}=3\right\rangle$ back to $|F=2\rangle$, corresponding to a projection of the atom onto $|F=2\rangle$. The SE pulse is long enough that we can assume the atom reaches the steady state (the coin pulse should not interfere with that) meaning that the effective SE rate $\gamma_{\text {eff }}$ is given by the natural line width times the steadystate population of $\left|F^{\prime}=3\right\rangle$ as follows [21],

$$
\gamma_{\mathrm{eff}}=\frac{\gamma}{2} \frac{I / I_{s}}{1+I / I_{s}}
$$

from which we get the probability of a SE event per pulse

$$
\rho=\gamma_{\mathrm{eff}} t_{\mathrm{SE}}
$$

Technically, $\gamma_{\text {eff }}$ and $\rho$ change during a single trajectory. The probability of the first decay overall and in each further decay has to be scaled down by a factor 2 since the atom will be either exactly or close to an equal superposition of the two ground states. So the given rate represents an upper limit, good only for a couple of microseconds after a SE event. We estimate $\rho \approx 0.35$ for a SE power of $3 \mu \mathrm{W}$ for our experimental system, as elaborated in Sec. IV.

Since the SE light is introduced $30 \mu$ s after the start of the coin, SE events will interrupt the coin pulse at random times, the partial action of the coin operator in between two events of time delay $t$ is

$$
e^{i \frac{\pi t}{4 T} \hat{\sigma}_{x}}=\left[\begin{array}{cc}
\cos \left(\frac{\pi t}{4 T}\right) & i \sin \left(\frac{\pi t}{4 T}\right) \\
i \sin \left(\frac{\pi t}{4 T}\right) & \cos \left(\frac{\pi t}{4 T}\right)
\end{array}\right],
$$

where $T$ is the total length of the coin pulse.

This means that the state $\psi$ of the internal degree of freedom at the end of the coin sequence is only determined by the time of the last SE event $t^{\prime} \in[0.29,0.58] \times T$ and thus given by

$$
\left|\psi_{\text {int }}\right\rangle=\cos \left[\frac{\pi\left(T-t^{\prime}\right)}{4 T}\right]|2\rangle+i \sin \left[\frac{\pi\left(T-t^{\prime}\right)}{4 T}\right]|1\rangle .
$$

Here $|1\rangle$ and $|2\rangle$ represent the two internal states, $\mid F=$ $\left.1, m_{F}=0\right\rangle$ and $\left|F=2, m_{F}=0\right\rangle$, respectively. Equation (4) clearly shows that SE creates an imbalance in the internal state of the atoms toward $|F=2\rangle$, which gets transferred to the populations and results in a biased momentum distribution (see our simulations in Fig. 1). The initial external states in momentum space are given by

$$
\left|\psi_{\text {ext }}\right\rangle=1 / \sqrt{2}\left(|n=0\rangle+e^{i \phi}|n=1\rangle\right) .
$$

Each SE event also affects the external degree of freedom by shifting the quasimomentum $q$ by a random amount. Contrary to SE induced by the kicking beams [12], the atom does not incur any recoil from the absorption of a photon from the SE beam due to its perpendicular alignment to the walk axis. Note that SE is not only a phase scrambling because it affects both the internal (projection on to hyperfine levels) and external (shifting of the quasimomentum) degrees of freedom. Possible heating during the experiment is taken into account during these simulations by considering an initial distribution of the quasimomentum, $\Delta_{q}$. This quasimomentum represents the distribution of the $\mathrm{BEC}$ and is selected to best represent the experimental conditions. Also, possible recoil from emission is taken into account in the theoretical model.

In our simulations, we draw up to three Poisson-distributed times and perform the partial coin operator from the largest time that is still inside the coin duration. We also add the corresponding amount of random recoil (here taken to be uniformly distributed). Typical simulation results for five-step quantum walks at two kicking strengths $k$ are shown in Fig. 1, which clearly show transitions from quantum walks to classic walks as the SE probability $\rho$ increases.

\section{EXPERIMENTAL PROCEDURES}

Each experimental sequence starts with a BEC of approximately $4 \times 10^{4}{ }^{87} \mathrm{Rb}$ atoms at the $\left|F=1, m_{F}=0\right\rangle$ state. The BEC is then subjected to Bragg, walk operator kicking, and microwave pulses. A schematic outlining of the pulse sequences is shown in Fig. 2. The walk operator kicking pulse and Bragg pulse are realized with the same two counterpropagating laser beams that intercept on the BEC, although the Bragg pulse has a longer duration to drive the BEC into the state $\left|\psi_{\text {ext }}\right\rangle=1 / \sqrt{2}\left(|n=0\rangle+e^{i \phi}|n=1\rangle\right)[10,22]$. We control populations of the two internal states $|1\rangle$ and $|2\rangle$ using the microwave (coin toss) pulses resonant with the $|1\rangle$ to $|2\rangle$ transition.

The standard QW of $i$ number of steps is created with a sequence of pulses described by the operator $\left(\hat{\mathbf{U}}_{\text {step }}\right)^{i}=$ $[\hat{\mathbf{T}} \hat{\mathbf{M}}(\pi / 2,-\pi / 2)]^{i-1}[\hat{\mathbf{T}} \hat{\mathbf{M}}(\pi / 2, \pi)]$. To ensure that the $\mathrm{QW}$ is symmetric the first coin pulse in the sequence is a Hadamard gate, which prepares the initial internal states as $\hat{\mathbf{M}}(\pi / 2, \pi)|1\rangle=1 / \sqrt{2}(|1\rangle+|2\rangle)$. For the standard QWs in our experiments an additional phase offset is applied to the coin microwave pulses to cancel out a global phase that acts on the QW due to the kicking pulses [10,23]. A QW that has the proper phase offset is referred to as phase compensated. 

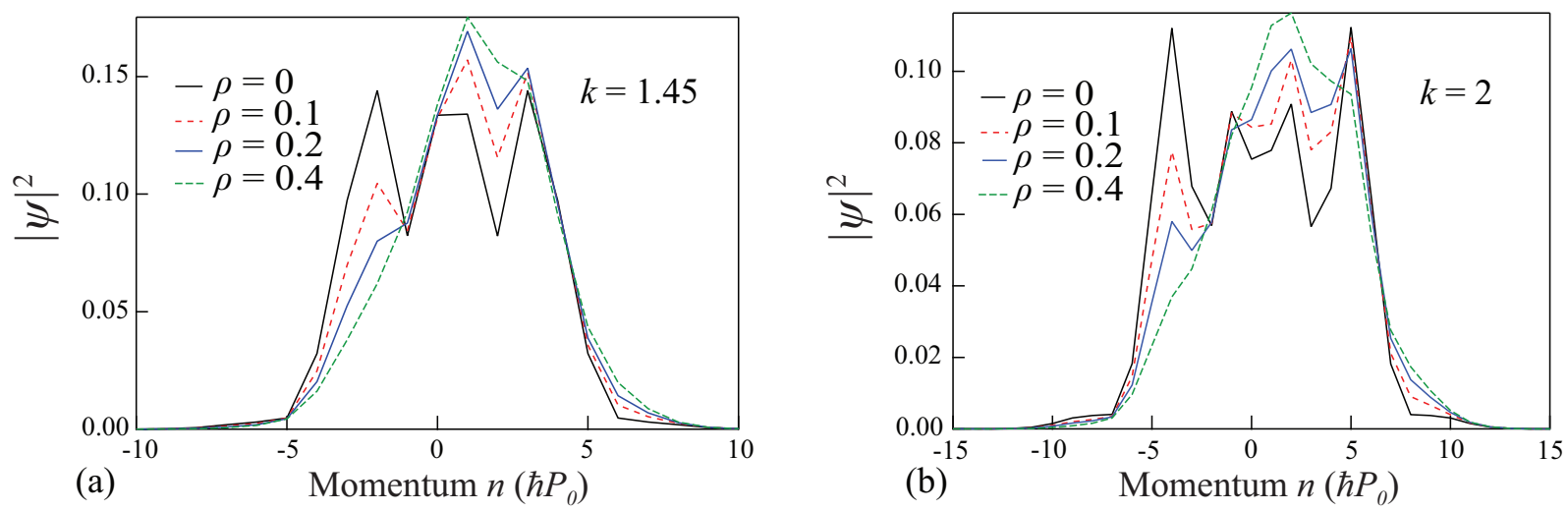

FIG. 1. Simulated momentum distributions of five-step quantum walks at two kicking strengths, $k=1.45$ (a) and $k=2.0$ (b), averaged over $10^{3}$ trajectories with $\Delta_{q}=0.025 \hbar P_{0}$ at various SE probability $\rho$. Here $\Delta_{q}$ is the width of the quasimomentum $q$. Note the increasing asymmetry as the probability $\rho$ increases.

During the coin toss pulses a SE light with a pulse duration of $30 \mu$ s is added to induce well-controlled SE effects onto the QW. A walk operator kicking pulse is then applied as the walk operator in momentum space followed by a coin toss microwave pulse. This coin toss pulse acts on the internal states to entangle the internal and external degrees of freedom. To avoid interfering with the walk operator kicking pulses, the SE pulses are set to apply only during the microwave coin pulses. This sequence of a coin toss pulse followed by a delta pulse is then repeated until QWs for $i$ number of steps are recorded and time-of-flight images are taken via the standard absorption imaging method [10,23].

\section{RESULTS AND DISCUSSIONS}

Figure 3 shows the effects of SE on a five-step QW in which the phase of the walk is noncompensated although the

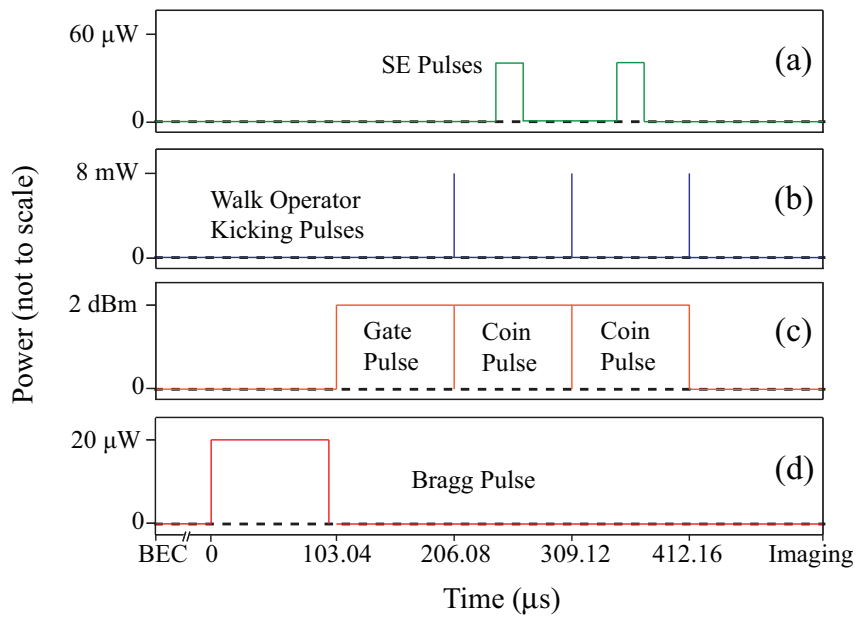

FIG. 2. Schematic diagram showing the sequence of various optical and microwave pulses used in our experiments. (a) The sequence of SE pulses each with a duration of $30 \mu \mathrm{s}$. (b) The sequence of the walk operator kicking pulses. (c) The sequence of the Hadamard gate and coin toss microwave pulses. The time duration of each of these pulses is $103.04 \mu \mathrm{s}$. (d) The sequence of the Bragg pulse with a duration of $103.04 \mu \mathrm{s}$. Axes are not to scale. phase of the microwave pulses is held constant throughout the data run. The walk operator kicking pulse strength during the noncompensated QWs is kept at $k=1.45$, which has been proved to yield ideal QWs $[10,23]$. The time-of-flight images shown in Fig. 3(a) indicate that the population of the atoms shifts toward the positive momentum states as the SE probability $\rho$ increases. This observation confirms the prediction of Fig. 1, i.e., SE creates an imbalance in the internal state of the atoms toward the $|2\rangle$ state leading to a biased momentum distribution because the projection of the $|2\rangle$ state moves in the direction of positive momenta. This shift in momentum is quantitatively analyzed in Fig. 4(c). In addition, the overall population of atoms present also decreases as $\rho$ increases. The effective decay rate of the QW is estimated from the observed exponential atom losses as $\rho$ increases [see the solid lines in Fig. 3(b) and Fig. 3(c)]. A typical example of our SE calibrations is shown in Fig. 3(c) which plots the condensate fraction of a BEC versus the duration of a single SE pulse at a fixed SE power of $3 \mu \mathrm{W}$. The BEC is first prepared in the $F=2$ state before being subjected to a SE pulse of light increasing in 50- $\mu$ s intervals. The exponential fitting of this data indicates that the probability of SE events at this power is $\rho=0.35$, as shown in Fig. 3(c).

We repeat the above experiment with a properly compensated QW generated at a higher kicking strength of $k \simeq 2$ to ensure that the QW distribution is broader than those created with the lower kicking strength. We scan the SE probability up to $\rho=0.84$ with an average of eight runs per power setting. The observed distribution of the QWs does not show noticeable differences beyond this $\rho$ value. Typical time-of-flight images of the compensated QWs are shown in Fig. 4(d), which indicate that the atoms in the compensated walks also shift toward the positive momentum states as the SE probability $\rho$ increases. The decay of the QW distribution can be more easily discerned from the momentum distributions, as displayed in Figs. 4(a) and 4(b). We also extract the mean momentum and mean energy from the noncompensated and compensated QW data, and respectively show them as a function of the SE probability $\rho$ in Fig. 4(c) and Fig. 4(e). A positive shift in the mean momentum as the SE pulses become more powerful is confirmed in Fig. 4(c) for both compensated and noncompensated QWs. In the case of the compensated QWs the mean 

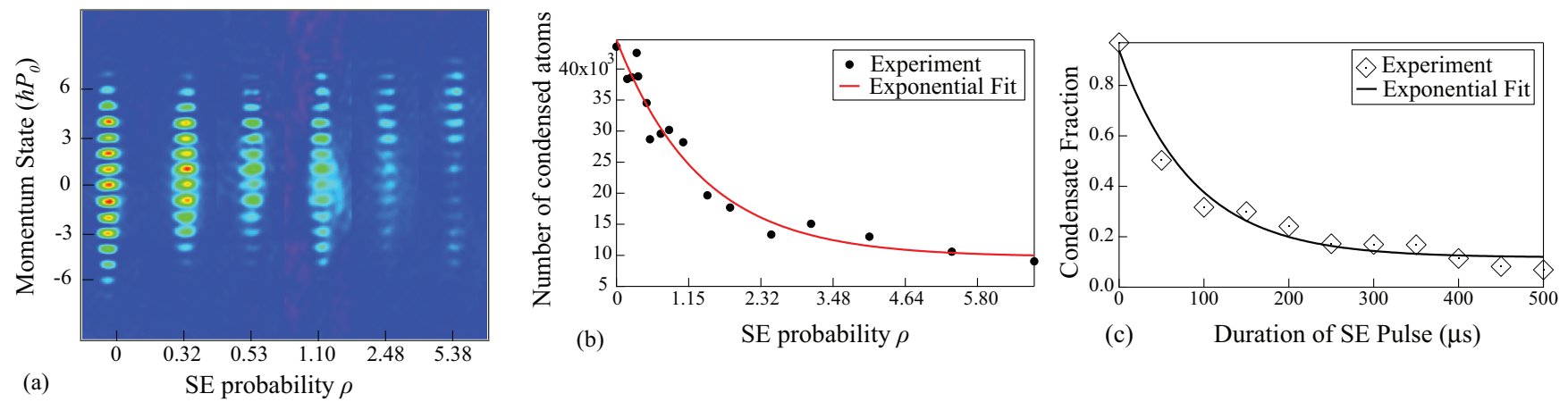

FIG. 3. (a) Time-of-flight images of a phase noncompensated five-step QW under various SE probability $\rho$ at the kicking strength $k=1.45$ and the SE pulse duration of $30 \mu \mathrm{s}$. (b) The number of condensed atoms versus $\rho$ in the noncompensated QWs shown in panel (a). (c) The condensate fraction versus SE pulse duration for a single BEC subjected to a pulse of SE light with a power of $3.0 \mu \mathrm{W}$. Solid lines in panel (b) and panel (c) are exponential fits (see text).

momentum is initially negative due to the phase of the applied microwave coin pulses being larger than $2 \pi$ thus causing an initial bias toward negative mean momentum. For a standard QW in our experiments this phase on the coin microwave pulses is normally below $(2 k+\pi)$ to cancel out a global phase that acts on the QW due to the kicking pulses [10,23]. Although this bias can adversely affect the momentum distribution of a QW that evolves in time, it does not prevent the observation of the positive shift in mean momentum as the SE probability $\rho$ increases. This is because the same microwave coin phase is applied throughout an experiment as the SE probability is scanned. On the other hand, Fig. 4(e) implies the mean energy remains constant for the compensated QWs as the SE probability $\rho$ increases within the range of 0 to 0.85 , which agrees with our simulations shown in Fig. 5(b).
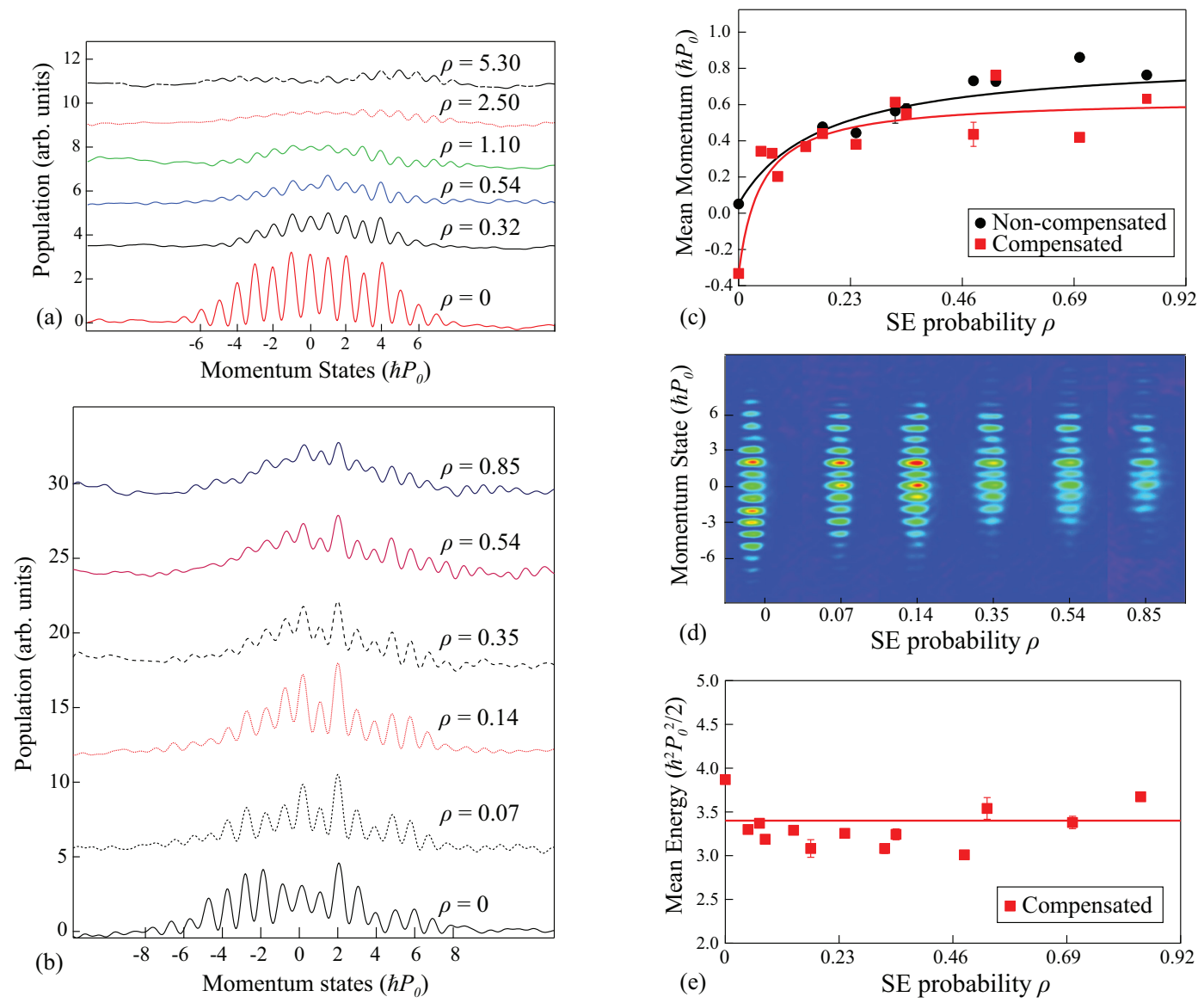

FIG. 4. Typical momentum distributions of (a) a phase noncompensated five-step QW and (b) a phase compensated five-step QW at various $\rho$ and at a fixed SE pulse duration of $30 \mu$ s (see text). Each momentum distribution is vertically shifted by a constant offset for visual clarity. (c) The mean momentum extracted from panels (a) and (b) as a function of $\rho$. (d) Typical time-of-flight images in the compensated QWs. (e) The mean energy extracted from panel (b) versus $\rho$. Solid lines in panel (c) and panel (e) are fitting curves to guide the eye. 

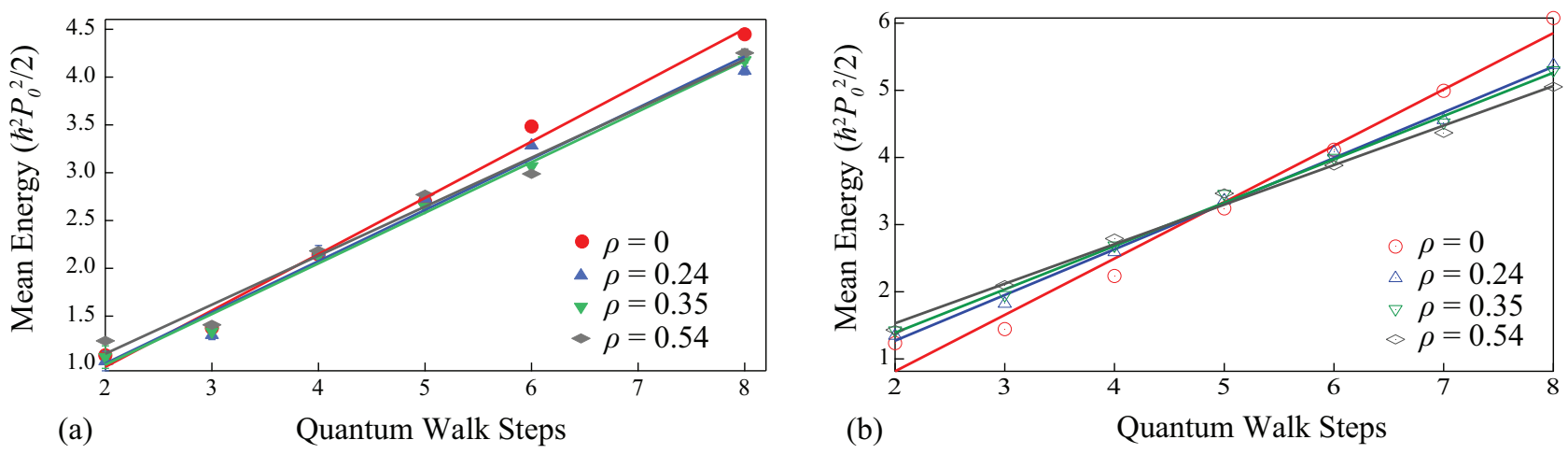

\begin{tabular}{|c||c|c|}
\hline SE Probability & Experimental Rate $R$ & Theoretical Rate $R$ \\
\hline \hline$\rho=0$ & 0.58 & 0.84 \\
$\rho=0.24$ & 0.53 & 0.68 \\
$\rho=0.35$ & 0.52 & 0.64 \\
$\rho=0.54$ & 0.51 & 0.58 \\
\hline
\end{tabular}

FIG. 5. (a) The extracted mean energy as a function of the QW steps at various SE probability $\rho$ and $k=1.4$. (See text.) (b) Theoretical predictions based on the experimental conditions for the data shown in panel (a). The solid lines in both panels represent the linear fitting functions. (c) Rate $R$ extracted from panel (a) and panel (b) (see text).

Our data in Fig. 3 and Fig. 4 indicate that the quantum to classical walk transitions happen at around $\rho=0.58$, much lower than the maximum SE probability studied in this paper. To clearly demonstrate the transition of walks from displaying quantum to classical behaviors under the applied SE pulses, we conduct similar experiments on walks of various numbers of steps. For each step, the kicking strength is kept at $k=1.4$ to reduce the probability of extra SE events induced by the kicking beams. The mean energy extracted from these experiments is plotted as a function of the QW steps for various $\rho$ in Fig. 5(a), which shows that the mean energy increases with increasing number of steps at a rate $R$. Each data set in Fig. 5(a) is fit with a linear function and the rate $R$ is calculated and tabulated in Fig. 5(c). The rate $R$ appears to increase as $\rho$ reduces and reach the largest value at $\rho=0$, as shown by the red markers in Fig. 5(a) and in Fig. 5(c). Our observations shown in Fig. 5 agree well with a predicted signature of quantum to classical walk transitions, i.e., QWs have larger mean energy than classical walks at a given step because QWs distribute ballistically while classical walks follow Gaussian distributions [1,10,11]. Figure 5 thus indicates that the QWs gradually transit to classical walks with less mean energy as the applied SE effect becomes powerful enough to destroy the entanglement of the two internal spin states. These results suggest that the rate $R$ could potentially be used as a good indicator for the quantum to classical walk transitions especially if only small numbers of walk steps are achievable. We also conduct theoretical simulations using similar parameters and typical simulation results are shown in Fig. 5(b) and Fig. 5(c). These simulations confirm that the rate $R$ reduces as the SE probability $\rho$ increases, leading to an increased difference in the mean energy among simulated data taken under various $\rho$ at a high-enough step, as clearly demonstrated by the six-step and eight-step data in Fig. 5(b). Good theory-experiment agreements demonstrated in Fig. 5 thus suggest that the rate $R$ can be an observable to quantify quantum to classical walk transitions. Figure 5 also shows that QWs are able to maintain their robustness when the number of steps is lower than five.

\section{CONCLUSIONS AND OUTLOOK}

We have presented quantum to classical walk transitions tuned by spontaneous emissions. The SE rate is derived from the observed atom losses during QWs. We have demonstrated that the addition of the SE light yields quantum to classical walk transitions and leads to biased momentum distributions, which can be well explained by our numerical simulations in both the compensated and noncompensated QWs. Our findings suggest a scheme to control the robustness of the quantum walks and demonstrate that the effects of the SE light are intrigue. While a SE event acts as a projective measurement on the internal spin degree of freedom, its effect on the external motion, that is the actually observed quantity, is direct by a change of the necessary resonance conditions for the QW, but also indirect since the motion becomes biased into the direction into which the ground state likes to move. Hence, for the center of mass of our atoms, SE is not a strong but rather a weak form of quantum measurement, with the internal state acting as an ancilla that is actually strongly measured. Many SE events will then necessarily have a larger effect than just one SE event since they bias more the walk into one direction of the external motion. Similar ideas have been put forward, e.g., in Ref. [24]. In conclusion, our results open further possibilities of utilizing the tunable SE light to engage on the theory of measurements in experimentally easily accessible quantum systems.

\section{ACKNOWLEDGMENT}

We thank the Noble Foundation for financial support. 
[1] J. Wang and K. Manouchehri, Physical Implementation of Quantum Walks (Springer, Berlin, 2014).

[2] W. Dur, R. Raussendorf, V. Kendon, and H.-J. Briegel, Quantum walks in optical lattices, Phys. Rev. A 66, 052319 (2002).

[3] K. Eckert, J. Mompart, G. Birkl, and M. Lewenstein, One- and two-dimensional quantum walks in arrays of optical traps, Phys. Rev. A 72, 012327 (2005).

[4] H. Schmitz, R. Matjeschk, C. Schneider, J. Glueckert, M. Enderlein, T. Huber, and T. Schaetz, Quantum Walk of a Trapped Ion in Phase Space, Phys. Rev. Lett. 103, 090504 (2009).

[5] M. Karsk, L. Forster, J. Choi et al., Quantum walk in position space with single optically trapped atoms, Science 325, 174 (2009).

[6] Y. Aharonov, L. Davidovich, and N. Zagury, Quantum random walks, Phys. Rev. A 48, 1687 (1993).

[7] A. Alberti and S. Wimberger, Quantum walk of a Bose-Einstein condensate in the Brillouin zone, Phys. Rev. A 96, 023620 (2017).

[8] C. M. Chandrashekar, Implementing the one-dimensional quantum (Hadamard) walk using a Bose-Einstein condensate, Phys. Rev. A 74, 032307 (2006).

[9] J. Kempe, Quantum random walks-an introductory overview, Contemp. Phys. 44, 307 (2003).

[10] S. Dadras, A. Gresch, C. Groiseau, S. Wimberger, and G. S. Summy, Experimental realization of a momentum-space quantum walk, Phys. Rev. A 99, 043617 (2019).

[11] S. Dadras, A. Gresch, C. Groiseau, S. Wimberger, and G. S. Summy, Quantum Walk in Momentum Space with a Bose-Einstein Condensate, Phys. Rev. Lett. 121, 070402 (2018).

[12] C. Groiseau and S. Wimberger, Spontaneous emission in quantum walks of a kicked Bose-Einstein condensate, Phys. Rev. A 99, 013610 (2019).
[13] N. Shenvi, J. Kempe, and K. Birgitta Whaley, Quantum randomwalk search algorithm, Phys. Rev. A 67, 052307 (2003).

[14] R. Portugal, Quantum Walks and Search Algorithms (Springer, Berlin, 2018).

[15] J. K. Asboth, Symmetries, topological phases, and bound states in the one-dimensional quantum walk, Phys. Rev. B 86, 195414 (2012).

[16] M. B. d'Arcy, R. M. Godun, G. S. Summy, I. Guarneri, S. Wimberger, S. Fishman, and A. Buchleitner, Decoherence as a probe of coherent quantum dynamics, Phys. Rev. E 69, 027201 (2004).

[17] M. B. d'Arcy, R. M. Godun, M. K. Oberlather, G. S. Summy, and K. Burnett, Approaching classicality in quantum accelerator modes through decoherence, Phys. Rev. E 64, 056233 (2001).

[18] R. K. Shrestha, J. Ni, W. K. Lam, G. S. Summy, and S. Wimberger, Dynamical tunneling of a Bose-Einstein condensate in periodically driven systems, Phys. Rev. E 88, 034901 (2013).

[19] S. Chai and M. F. Andersen, Enhancing survival resonances with engineered dissipation, Phys. Rev. Research 2, 033194 (2020).

[20] S. Chai, J. Fekete, and M. F. Andersen, Measuring the local gravitational field using survival resonances in a dissipatively driven atom-optics system, Phys. Rev. A 98, 063614 (2018).

[21] Daniel A. Steck, Rubidium 87 D Line Data, available online at http://steck.us/alkalidata (revision 2.2.2, 9 July 2021).

[22] Y. Torii, Y. Suzuki, M. Kozuma, T. Sugiura, T. Kuga, L. Deng, and E. W. Hagley, Mach-Zehnder Bragg interferometer for a Bose-Einstein condensate, Phys. Rev. A 61, 041602(R) (2000).

[23] S. Dadras, Discrete-time quantum walk of a Bose-Einstein condensate in momentum space, Ph.D. thesis, Oklahoma State University, 2018.

[24] B. Tamir and E. Cohen, Introduction to weak measurements and weak values, Quanta 2, 7 (2013). 